\title{
On the Existence of a Codimension 1 Completely Integrable Totally Geodesic Distribution on a Pseudo-Riemannian Heisenberg Group
}

\author{
Wafaa BATAT $^{\dagger}$ and Salima RAHMANI ${ }^{\ddagger}$ \\ † Ecole Normale Supérieure de L'Enseignement Technologique d'Oran, \\ Département de Mathématiques et Informatique, B.P. 1523 El M'Naouar Oran, Algeria \\ E-mail:wafa.batat@enset-oran.dz,wafa.batat@usc.es \\ ¥ Laboratoire de Mathématiques-Informatique et Applications, Université de Haute Alsace, \\ 68093 Mulhouse Cedex, France \\ E-mail: ramses161616@yahoo.fr \\ $\S$ Ecole Doctorale de Systèmes Dynamiques et Géométrie, Département de Mathématiques, \\ Faculté des Sciences, Université des Sciences et de la Technologie d'Oran, \\ B.P. 1505 Oran El M'Naouer, Algeria
}

Received December 23, 2009, in final form February 23, 2010; Published online February 28, 2010 doi:10.3842/SIGMA.2010.021

\begin{abstract}
In this note we prove that the Heisenberg group with a left-invariant pseudoRiemannian metric admits a completely integrable totally geodesic distribution of codimension 1. This is on the contrary to the Riemannian case, as it was proved by T. Hangan.

Key words: Heisenberg group; pseudo-Riemannian metrics; geodesics; codimension 1 distributions; completely integrable distributions
\end{abstract}

2010 Mathematics Subject Classification: 58A30; 53C15; 53C30

\section{Introduction}

Given an $n$-dimensional $C^{\infty}$ manifold $M$, a distribution $D$ of codimension $k$ of $M$ is an $(n-k)$ dimensional subbundle of the tangent space $T M$. A distribution $D$ of a pseudo-Riemannian manifold $(M, g)$ is called totally geodesic if every geodesic tangent to $D$ at some point remains everywhere tangent to $D$. Among 2-step nilpotent Lie groups with left-invariant metrics, the Heisenberg group is of particular significance. In [8], the second author proved that there are three nonisometric left-invariant Lorentzian metrics on the 3-dimensional Heisenberg group, one of which is flat. This is a strong contrast to the Riemannian case in which there is only one (up to positive homothety) and it is not flat.

Another major difference to the Riemannian case appears. Indeed, T. Hangan proved that the Heisenberg group endowed with a left-invariant Riemannian metric does not admit any codimension 1 completely integrable totally geodesic distribution (see [5] for the three-dimensional case and [6] for the higher dimensional case). Recently, in [9] the second author and N. Rahmani proved that the 3-dimensional Heisenberg group admits a left-invariant Lorentzian metric for which there exists a codimension 1 completely integrable totally geodesic distribution. It is an interesting problem to investigate whether and to what extent results valid in Riemannian Heisenberg group can be extended to the pseudo-Riemannian case. In fact, in a high-dimensional Heisenberg group there are different types of pseudo-Riemannian metrics while there is still essentially only one (up to positive homothety) Riemannian metric. So, the purpose of this paper is to prove, contrary to the Riemannian case, the existence of a codimension 1 completely in- 
tegrable totally geodesic distribution on a pseudo-Riemannian Heisenberg group of dimension $2 p+1$.

\section{Geodesics and totally geodesic distributions on Heisenberg group}

Consider $\mathbb{R}^{2 p+1}$ with the elements of the form $X=\left(x_{1}, \ldots, x_{p}, y_{1}, \ldots, y_{p}, z\right)$. Define the product on $\mathbb{R}^{2 p+1}$, by

$$
X \tilde{X}=\left(x_{1}+\tilde{x}_{1}, \ldots, x_{p}+\tilde{x}_{p}, y_{1}+\tilde{y}_{1}, \ldots, y_{p}+\bar{y}_{p}, z+\tilde{z}-\sum_{i=1}^{p} x_{i} \tilde{y}_{i}\right),
$$

where $X=\left(x_{1}, \ldots, x_{p}, y_{1}, \ldots, y_{p}, z\right), \tilde{X}=\left(\tilde{x}_{1}, \ldots, \tilde{x}_{p}, \tilde{y}_{1}, \ldots, \tilde{y}_{p}, \tilde{z}\right)$.

Let $H_{2 p+1}=\left(\mathbb{R}^{2 p+1}, g\right)$ be the $(2 p+1)$-dimensional Heisenberg group with the left-invariant pseudo-Riemannian metric $g$ defined by

$$
g=-\sum_{i=1}^{p}\left(d x_{i}\right)^{2}+\sum_{i=1}^{p}\left(d y_{i}\right)^{2}+\left(d z+\sum_{i=1}^{p} x_{i} d y_{i}\right)^{2} .
$$

With respect to the basis of coordinate vector fields $\left\{\partial_{x_{i}}=\frac{\partial}{\partial x_{i}}, \partial_{y_{i}}=\frac{\partial}{\partial y_{i}}, \partial_{z}=\frac{\partial}{\partial z}\right\}, i=$ $1,2, \ldots, p$ for which (1) holds, the nonvanishing metric components are

$$
\begin{aligned}
& g_{\partial x_{i} \partial x_{i}}=-1, \quad g_{\partial y_{i} \partial y_{i}}=1+\left(x_{i}\right)^{2}, \quad g_{\partial y_{i} \partial z}=x_{i} \quad \text { for } \quad 1 \leq i \leq p, \\
& g_{\partial_{z} \partial_{z}}=1, \quad g_{\partial y_{i} \partial_{y_{j}}}=x_{i} x_{j} \quad \text { for } \quad 1 \leq i \neq j \leq p .
\end{aligned}
$$

The geodesics equations are obtained in [2] (for the Riemannian case) and in [1] and [4] (for the pseudo-Riemannian case), in more general context of two-step nilpotent Lie groups with a left-invariant metric. Jang and Parker [7] later gave explicit formulas for geodesics on the 3dimensional Heisenberg group endowed with a left-invariant Lorentzian metric. A corresponding description was made in [5] in the Riemannian case.

Here we will find the geodesics equations of $H_{2 p+1}$ for the left-invariant pseudo-Riemannian metric (1) by integrating their Euler-Lagrange equations. Notice that Hangan obtained in [6] all geodesics of the Riemannian Heisenberg group of dimension $2 p+1$.

So, let $\gamma(t)$ be a geodesic curve on $H_{2 p+1}$, determined, with respect to the basis of coordinate vector fields $\left\{\frac{\partial}{\partial x_{1}}, \ldots, \frac{\partial}{\partial x_{p}}, \frac{\partial}{\partial y_{1}}, \ldots, \frac{\partial}{\partial y_{p}}, \frac{\partial}{\partial z}\right\}$, by its components $(x(t), y(t), z(t))$ where $x(t)=$ $\left(x_{1}(t), \ldots, x_{p}(t)\right), y(t)=\left(y_{1}(t), \ldots, y_{p}(t)\right)$ and $z(t) \in \mathbb{R}$. Using $(2)$, the Lagrangian associated to the metric (1) is

$$
L=\frac{1}{2}\left\{-\sum_{i=1}^{p}\left(x_{i}^{\prime}\right)^{2}+\sum_{i=1}^{p}\left(y_{i}^{\prime}\right)^{2}+\left[z^{\prime}+\sum_{i=1}^{p} x_{i} y_{i}^{\prime}\right]^{2}\right\}
$$

with $x^{\prime}(t)=\left(x_{1}^{\prime}(t), \ldots, x_{p}^{\prime}(t)\right)$ and $y^{\prime}(t)=\left(y_{1}^{\prime}(t), \ldots, y_{p}^{\prime}(t)\right)$. Hence, the corresponding EulerLagrange equations are

$$
\begin{aligned}
& x^{\prime \prime}+\left(z^{\prime}+\sum_{i=1}^{p} x_{i} y_{i}^{\prime}\right) y^{\prime}=0, \quad y^{\prime \prime}+\left(z^{\prime}+\sum_{i=1}^{p} x_{i} y_{i}^{\prime}\right) x^{\prime}=0 \\
& z^{\prime \prime}+\sum_{i=1}^{p}\left(x_{i}^{\prime} y_{i}^{\prime}+x_{i} y_{i}^{\prime \prime}\right)=0
\end{aligned}
$$


and we will integrate the system (3) with the initial conditions

$$
\begin{array}{lll}
x(0)=x_{0}, & y(0)=y_{0}, & z(0)=z_{0}, \\
x^{\prime}(0)=x_{0}^{\prime}, & y^{\prime}(0)=y_{0}^{\prime}, & z^{\prime}(0)=z_{0}^{\prime} .
\end{array}
$$

The first integration of the last equation of the Euler-Lagrange equations, reduces the system (3) to

$$
x^{\prime \prime}+\alpha y^{\prime}=0, \quad y^{\prime \prime}+\alpha x^{\prime}=0, \quad z^{\prime}+\sum_{i=1}^{p} x_{i} y_{i}^{\prime}=\alpha,
$$

where $\alpha=z_{0}^{\prime}+\sum_{i=1}^{p} x_{i}(0) y_{i}^{\prime}(0)$ is a real constant. For the system (4) we distinguish two cases.

If $\alpha=0$, the geodesics curves are given by

$$
\begin{aligned}
& x(t)=x_{0}^{\prime} t+x_{0}, \quad y(t)=y_{0}^{\prime} t+y_{0}, \\
& z(t)=z_{0}-\sum_{i=1}^{p}\left(x_{i}^{\prime}(0) \frac{t}{2}+x_{i}(0)\right) y_{i}^{\prime}(0) t .
\end{aligned}
$$

If $\alpha \neq 0$, the geodesics curves are given by

$$
\begin{aligned}
x(t)= & \frac{1}{\alpha}\left(\sinh (\alpha t) x_{0}^{\prime}-\cosh (\alpha t) y_{0}^{\prime}+\alpha x_{0}+y_{0}^{\prime}\right), \\
y(t)= & \frac{1}{\alpha}\left(-\cosh (\alpha t) x_{0}^{\prime}+\sinh (\alpha t) y_{0}^{\prime}+\alpha y_{0}+x_{0}^{\prime}\right), \\
z(t)= & \frac{1}{\alpha^{2}} \sum_{i=1}^{p}\left(\alpha x_{i}(0)+y_{i}^{\prime}(0)\right)\left(x_{i}^{\prime}(0) \cosh (\alpha t)-y_{i}^{\prime}(0) \sinh (\alpha t)\right) \\
& -\frac{1}{2 \alpha^{2}} \sum_{i=1}^{p} x_{i}^{\prime}(0) y_{i}^{\prime}(0) \cosh (2 \alpha t) \\
& +\frac{1}{4 \alpha^{2}} \sum_{i=1}^{p}\left[\left(x_{i}^{\prime}(0)\right)^{2}+\left(y_{i}^{\prime}(0)\right)^{2}\right] \sinh (2 \alpha t)+\left(\alpha-\frac{1}{2 \alpha} \sum_{i=1}^{p}\left[\left(x_{i}^{\prime}(0)\right)^{2}-\left(y_{i}^{\prime}(0)\right)^{2}\right]\right) t \\
& +z_{0}-\frac{1}{2 \alpha^{2}} \sum_{i=1}^{p} x_{i}^{\prime}(0)\left(2 \alpha x_{i}(0)+y_{i}^{\prime}(0)\right) .
\end{aligned}
$$

It must be noted that the pseudo-Riemannian metric $g$ on a $(2 p+1)$-dimensional Heisenberg group is geodesically complete. This is a general truth for pseudo-Riemannian 2-step nilpotent Lie groups. In fact, in [3] Guediri proved that every left-invariant pseudo-Riemannian metric on a 2-step nilpotent Lie groups is geodesically complete.

We now prove the following result

Theorem 1. The pseudo-Riemannian metric (1) admits a codimension 1 completely integrable totally geodesic distribution on the Heisenberg group $H_{2 p+1}$.

Proof. Let $\gamma$ be a geodesic curve on $H_{2 p+1}$ with $\gamma(0)=\left(x_{0}, y_{0}, z_{0}\right)$ and $\gamma^{\prime}(0)=\left(x_{0}^{\prime}, y_{0}^{\prime}, z_{0}^{\prime}\right)$. Consider in $H_{2 p+1}$ the 1 -form $\theta=\sum_{i=1}^{p}\left(d x_{i}-d y_{i}\right)$.

If $z_{0}^{\prime}+\sum_{i=1}^{p} x_{i}(0) y_{i}^{\prime}(0)=0$ then $\gamma$ is given by (5) and we have

$$
x^{\prime}(t)=x_{0}^{\prime}, \quad y^{\prime}(t)=y_{0}^{\prime} .
$$




$$
\begin{aligned}
& \text { If } z_{0}^{\prime}+\sum_{i=1}^{p} x_{i}(0) y_{i}^{\prime}(0) \neq 0 \text { then } \gamma \text { is given by (6) and we have } \\
& \qquad x_{i}^{\prime}(t)=\left(\cosh (\alpha t) x_{i}^{\prime}(0)-\sinh (\alpha t) y_{i}^{\prime}(0)\right), \quad y_{i}^{\prime}(t)=\left(-\sinh (\alpha t) x_{i}^{\prime}(0)+\cosh (\alpha t) y_{i}^{\prime}(0)\right)
\end{aligned}
$$

for all $i \in\{1,2, \ldots, p\}$. From (5) and (6) one gets

$$
\begin{aligned}
& \theta\left(\gamma^{\prime}(t)\right)=\sum_{i=1}^{p}\left(x_{i}^{\prime}(0)-y_{i}^{\prime}(0)\right) \quad \text { if } \quad z_{0}^{\prime}+\sum_{i=1}^{p} x_{i}(0) y_{i}^{\prime}(0)=0, \\
& \theta\left(\gamma^{\prime}(t)\right)=[\cosh (\alpha t)+\sinh (\alpha t)]\left(\sum_{i=1}^{p}\left(x_{i}^{\prime}(0)-y_{i}^{\prime}(0)\right)\right) \quad \text { if } \quad z_{0}^{\prime}+\sum_{i=1}^{p} x_{i}(0) y_{i}^{\prime}(0) \neq 0,
\end{aligned}
$$

for all $t \in \mathbb{R}$. This means that, in both cases, if $\theta\left(\gamma^{\prime}(0)\right)=0$ then $\theta\left(\gamma^{\prime}(t)\right)=0$ for all $t \in \mathbb{R}$. In other words, the kernel of the given 1 -form $\theta$ defines a codimension 1 totally geodesic distribution. Since $\theta$ is closed, the distribution $D=\operatorname{ker} \theta$ is involutive and from the Frobenius theorem it follows that it is completely integrable.

Notice that since $\theta$ is the differential of the function $f=\sum_{i=1}^{p}\left(x_{i}-y_{i}\right)$, the submanifolds given by $f=$ const are the maximal integral submanifolds of $D$.

Remark. Since the dimension of the center of a Heisenberg group is 1 , it seems natural to pose the study of the existence of totally geodesic distributions on 2-step nilpotent Lie groups endowed with a pseudo-Riemannian metric with index equal to the dimension of the center of the Lie algebra.

\section{Acknowledgements}

The authors wish to express their gratitude toward the referees for their valuable remarks and also would like to thank Professor José Antonio Oubiña for his fruitful comments on the revised version of this paper. The first author was supported by ENSET d'Oran and the second author is indebted to Doctoral School of Dynamical Systems and Geometry (U.S.T. Oran) for its financial support during the elaboration of this work.

\section{References}

[1] Cordero L.A., Parker P.E., Pseudoriemannian 2-step nilpotent Lie groups, math.DG/9905188.

[2] Eberlein P., Geometry of 2-step nilpotent groups with a left invariant metric, Ann. Sci. École Norm. Sup. (4) 27 (1994), 611-660.

[3] Guediri M., Sur la complétude des pseudo-métriques invariantes a gauche sur les groupes de Lie nilpotents, Rend. Sem. Mat. Univ. Politec. Torino 52 (1994), 371-376.

[4] Guediri M., Lorentz geometry of 2-step nilpotent Lie groups, Geom. Dedicata 100 (2003), 11-51.

[5] Hangan T., Au sujet des flots riemanniens sur le groupe nilpotent de Heisenberg, Rend. Circ. Mat. Palermo (2) 35 (1986), 291-305.

[6] Hangan T., Sur les distributions totalement géodésiques du groupe nilpotent riemannien $H_{2 p+1}$, Rend. Sem. Fac. Sci. Univ. Cagliari 55 (1985), 31-47.

[7] Jang C., Parker P.E., Examples of conjugate loci of pseudoRiemannian 2-step nilpotent Lie groups with nondegenerate center, in Recent Advances in Riemannian and Lorentzian Geometries (Baltimore, MD, 2003), Editors K.L. Duggal and R. Sharma, Contemp. Math., Vol. 337, Amer. Math. Soc., Providence, RI, 2003, 91-108.

[8] Rahmani S., Métriques de Lorentz sur les groupes de Lie unimodulaires, de dimension 3, J. Geom. Phys. 9 (1992), 295-302.

[9] Rahmani N., Rahmani S., Lorentzian geometry of the Heisenberg group, Geom. Dedicata 118 (2006), 133140. 